\title{
REVIEW
}

\section{Constrictive pericarditis in the modern era: a diagnostic dilemma}

\author{
R A Nishimura
}

Constrictive pericarditis is caused by fibrosis and calcification of the pericardium, processes that inhibit diastolic filling of the heart. This condition has posed a diagnostic dilemma since it was first recognised clinically. ${ }^{1}$ Although many diagnostic approaches have become available subsequently, the diagnostic challenge remains. ${ }^{2}$ Because surgical intervention can provide complete relief of symptoms in many patients, accurate diagnosis of this disorder is important. ${ }^{3}$

In the past, it was necessary to differentiate constrictive pericarditis from other causes of right sided heart failure, such as pulmonary embolism, pulmonary hypertension, right ventricular infarction, mitral stenosis, and left ventricular systolic dysfunction. Now, with two dimensional and Doppler echocardiography, these other causes of right sided heart failure can be diagnosed or excluded. Imaging methods such as computed tomography and magnetic resonance scanning can measure pericardial thickness, which is usually increased in patients with constrictive pericarditis. ${ }^{4}$ However, the aetiology of constrictive pericarditis has changed during the past few decades, leading to further diagnostic uncertainties. ${ }^{3}$ In the past, many patients with constrictive pericarditis had severe calcification of the entire pericardium, usually secondary to tuberculous pericarditis. Today, other causes of constrictive pericarditis are common, such as mantle chest radiation and open heart surgical procedures. Diffuse calcification of the pericardium occurs much less commonly in these patients with constrictive pericarditis and the pericardial thickness may even be normal. After radiation therapy or an open heart operation, constrictive pericarditis, myocardial restrictive disease, or a combination of both may develop. ${ }^{3}$

Thus, patients today have signs and symptoms of right sided heart failure that are disproportionate to left ventricular dysfunction or valvar disease. The challenge is to determine whether abnormalities are caused by pericardial restraint, myocardial restriction, or both. ${ }^{256}$ If pericardial and myocardial disease are present, the decision to proceed with the operation depends on the degree to which the abnormal pericardium contributes to the increased intracardiac pressures. Previous diagnostic studies, including cardiac catheterisation, have not been able to make this determination. ${ }^{5-7}$ Insights into the pathophysiology of constrictive and restrictive disorders have emerged, describing respiratory changes in pressures and flows. ${ }^{7-9}$ To meet the diagnostic challenge, non-invasive and invasive diagnostic testing must frequently be informed by a knowledge of pathophysiological mechanisms.

\section{Pathophysiology}

In constrictive pericarditis and restrictive cardiomyopathy, ventricular filling is restricted. ${ }^{25}{ }^{10}$ In the former, a rigid pericardium restricts inflow into the ventricles after an initial expansion of the myocardium. In the latter, a stiff myocardium causes a major decrease in the effective operative compliance of the heart muscle itself. ${ }^{11}{ }^{12}$ In both conditions, a high driving pressure across the valves at the time of atrioventricular valve opening results in early rapid diastolic filling and an abrupt increase in ventricular pressure. When ventricular pressure increases to the point at which it exceeds atrial pressure, flow is terminated in early diastole. The end result is a major increase of diastolic pressures in all four cardiac chambers, with a dip and plateau pattern seen on the ventricular pressure curves and a restriction to filling pattern seen on the transmitral Doppler flow velocity curves.

As reported in 1989 by Hatle and colleagues, ${ }^{9}$ dynamic changes with respiration occur in patients with constrictive pericarditis but not in patients with restrictive cardiomyopathy. These changes are, firstly, dissociation of intrathoracic and intracardiac pressures and, secondly, enhanced ventricular interaction. Dissociation of intrathoracic and intracardiac pressures results in respiratory shifts in the diastolic driving pressure across the mitral valve. In the heart with a normal pericardium, inspiration causes a decrease in intrathoracic pressure, which is reflected in the cardiac chambers. Thus, during the respiratory cycle, there is no change in the driving pressure from the lungs across the pulmonary veins, into the left atrium, and across the mitral valve into the left ventricle. However, in a patient with constrictive pericarditis, the rigid pericardium does not allow the decrease in intrathoracic pressure to be transmitted to the left sided chambers. During inspiration there is a lower driving force from the lungs into the left side of the heart and the left ventricle becomes underfilled. 


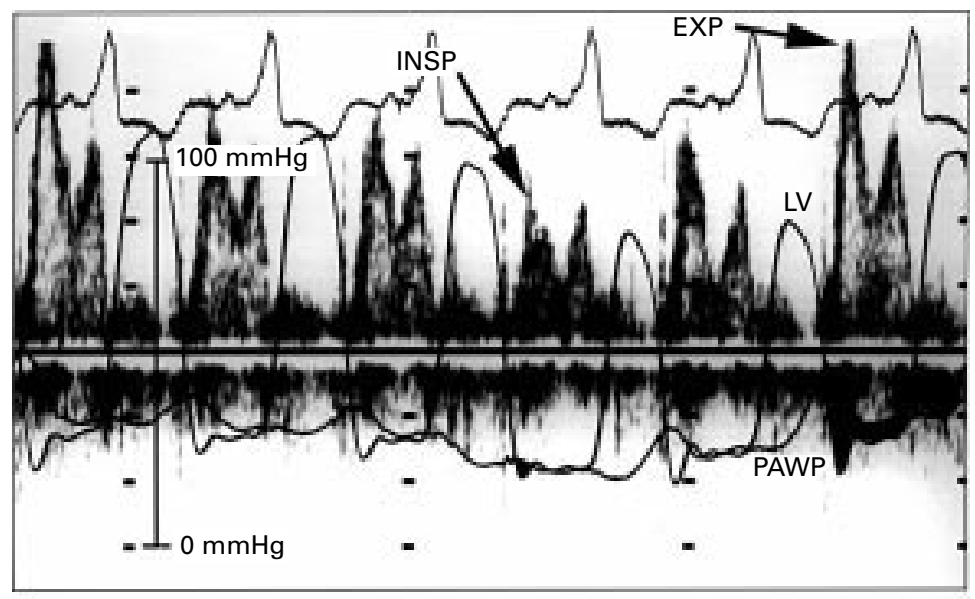

Figure 1 Transmitral flow velocity in a patient with constrictive pericarditis. During peak inspiration (INSP), there is a decrease in the early diastolic driving pressure across the mitral valve, seen as the initial gradient between the pressure in the left ventricle (LV) and the pulmonary artery wedge pressure (PAWP). This results in a decrease in the initial $E$ velocity on the transmitral flow velocity curve. During expiration (EXP), there is an increase in the transmitral gradient between the pressure in the LV and the PAWP, resulting in an increase in the initial $E$ velocity and the transmitral flow velocity curve. Data were obtained simultaneously by Doppler echocardiography and high fidelity manometer tipped catheters.

Enhanced ventricular interaction is another effect of pericardial restraint of the cardiac chambers. In a patient with constrictive pericarditis, during inspiration the left ventricle is underfilled and there is a reciprocal increase in filling of the right ventricle. Conversely, during expiration there is decreased filling of the right ventricle and increased filling of the left ventricle. In a patient with a normal pericardium, during inspiration there is some increase in filling of the right ventricle because of enhanced venous return but filling of the left ventricle is unaffected throughout the cardiac cycle. These dynamic changes throughout the respiratory cycle can be used to differentiate constrictive pericarditis from restrictive cardiomyopathy.

\section{Two dimensional and Doppler echocardiography}

Two dimensional echocardiography is useful in the diagnosis of constrictive pericarditis. It is used mainly to rule out other causes of right sided heart failure, such as left ventricular systolic dysfunction, unsuspected mitral valve disease, and pulmonary hypertension. Severe biatrial enlargement and thickened ventricular walls with an unusual texture are classic findings of two dimensional echocardiography in cases of restrictive or infiltrative cardiomyopathy. ${ }^{12}$ These findings, although specific for a restrictive cardiomyopathy, are usually not present in most patients with constrictive pericarditis. In a patient with severe classic constrictive pericarditis, an unusual septal bounce occurs from the interaction of the left and right ventricular diastolic pressures and is accompanied by an inspiratory septal shift. ${ }^{13}$ Although it is unusual for constrictive pericarditis to occur in the absence of septal abnormalities, such findings cannot be considered diagnostic. Assessment of pericardial thickness is difficult on transthoracic echocardiographic imaging. ${ }^{14}$

Doppler echocardiography provides additional diagnostic information in cases of constrictive pericarditis. ${ }^{89}$ Doppler interrogation of transmitral flow, pulmonary vein flow, and hepatic vein flow is used widely to analyse diastolic filling of the heart. ${ }^{15}$ The pathophysiological features of constrictive pericarditis and restrictive cardiomyopathy described above can be evaluated by Doppler flow patterns. The transmitral flow velocity curve, assessed with a pulsed wave sample volume placed at the tip of the mitral valve leaflets, indicates the driving pressure across the mitral valve. The contour reflects left atrial pressure, left atrial compliance, left ventricular compliance, and the rate of ventricular relaxation. ${ }^{15}$ In patients with early rapid restricted filling, there is a high initial $\mathrm{E}$ velocity, a short deceleration of the initial flow, and a reduced velocity at atrial contraction. This transmitral flow velocity contour is seen in patients with constrictive pericarditis and in those with restrictive cardiomyopathy.

Respiratory changes in the transmitral flow velocity curve may differentiate patients with constrictive pericarditis from those with restrictive cardiomyopathy (fig 1). ${ }^{9}$ In patients with constrictive pericarditis, the dissociation of intrathoracic and intracardiac pressures results in a decrease in initial driving pressure into the left ventricle during inspiration. Consequently, during the first beat of inspiration, there is a decrease in the initial $\mathrm{E}$ velocity on the transmitral flow velocity curve. During expiration, there is a pronounced increase in the initial E velocity. In patients with restrictive cardiomyopathy and in others with a normal pericardium, there is no inspiratory change in the initial E velocity because there is little change in the driving pressure into the left ventricle throughout the respiratory cycle. The same respiratory variation is seen in the diastolic forward flow velocities when the pulmonary venous inflow is interrogated. Reciprocal changes occurring in the right ventricle during respiration can be assessed by analysis of tricuspid and hepatic vein flows.

Although Doppler interrogation of transmitral flow and pulmonary venous flow is useful for confirming the diagnosis of constrictive pericarditis, Doppler findings can be misleading in some instances. In patients with irregular rhythms such as atrial fibrillation, the varying RR intervals may themselves cause changes in the initial $\mathrm{E}$ velocity on the transmitral flow velocity curve. Large respiratory variations in intrathoracic pressure, such as those seen in patients with chronic obstructive pulmonary disease, may result in an inspiratory decrease in the initial $\mathrm{E}$ velocity, simulating the changes seen in patients with constrictive pericarditis. In a patient with constrictive pericarditis, there may be no change in the mitral inflow velocity curve if the filling pressure is greatly increased because the relative inspiratory changes in the driving pressure across the mitral valve are overshadowed by the high initial driving pressure. $^{16}$

A comprehensive two dimensional and Doppler echocardiogram may be considered diagnostic in a subset of patients with classic constrictive pericarditis. In patients with a clinical diagnosis consistent with constrictive 

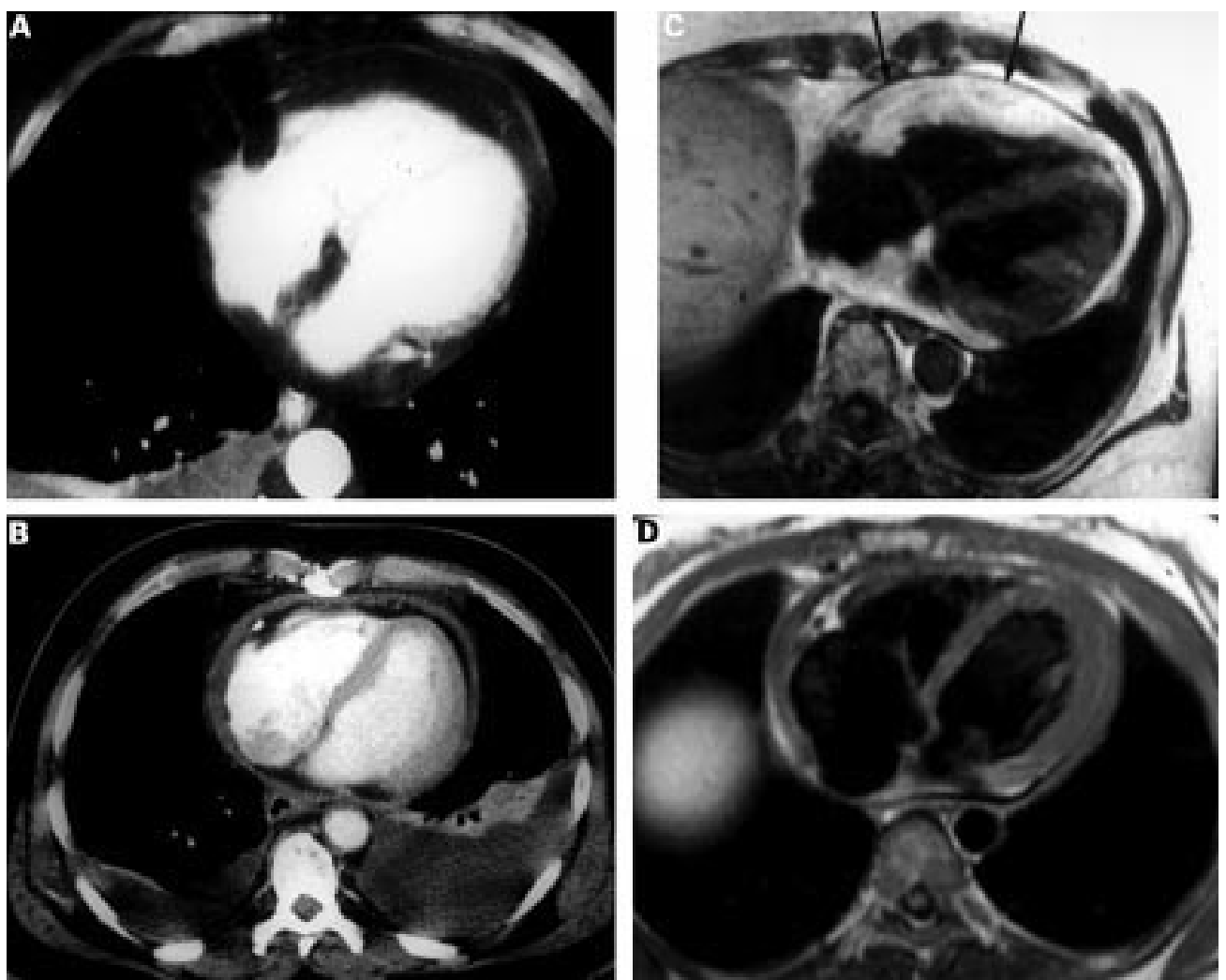

Figure 2 Normal and abnormal pericardium seen on computed tomography (CT) and magnetic resonance imaging (MRI) studies. (A) CT scan of normal pericardium. (B) CT scan of thickened pericardium. (C) MRI scan of normal thick pericardium (arrows). (D) MRI scan of a thickened pericardium. Reproduced from Breen ${ }^{18}$ with permission of Lippincott Williams $\mathcal{E}$ Wilkins.

pericarditis, echocardiographic findings can confirm the clinical suspicion if the two dimensional echocardiogram shows a septal bounce and respiratory septal shift and there are inspiratory decreases in the initial $\mathrm{E}$ velocity on the mitral inflow velocity curve. However, equivocal echocardiographic findings may be present in up to one third of patients with possible pericardial constriction, especially those with mixed pericardial and myocardial disease. Further testing is required in such patients.

\section{Computed tomography and magnetic resonance imaging}

Direct visualisation of the pericardium may be helpful in the diagnosis of constrictive pericarditis. Although pericardial thickening can be detected by transoesophageal echocardiography, ${ }^{14}$ the standard imaging methods used have been computed tomography and magnetic resonance imaging (fig 2). ${ }^{2517} 18$ Each of these methods can directly visualise the pericardium and detect an increase in pericardial thickness greater than $2 \mathrm{~mm}$ in diameter. These methods also yield other anatomical findings, such as distortion of the cardiac chambers by the pericardium, that can aid in diagnosis.

However, the finding of a thickened pericardium is not necessarily diagnostic of constrictive pericarditis. Some patients may have pericardial thickening but no evidence of constriction. For example, some degree of pericardial reaction may be present without a haemodynamic effect in patients who have had radiation therapy or an open heart operation.
Nor does the finding of normal pericardial thickness necessarily rule out a diagnosis of constrictive pericarditis. In up to $20 \%$ of patients with surgically confirmed constrictive pericarditis, imaging methods may show a pericardium of normal thickness. In such patients, the constrictive process may be caused by epicardial constriction rather than pericardial constriction.

\section{Cardiac catheterisation}

Cardiac catheterisation can yield findings that are classic for constrictive pericarditis. ${ }^{10} 1920$ These findings include an increase and equalisation of end diastolic pressures in all four cardiac chambers, a dip and plateau pattern in the ventricular pressure curves, and rapid $\mathrm{x}$ and $\mathrm{y}$ descents in the atrial pressure curves. However, these findings also may be present in patients with restrictive cardiomyopathy. Several studies have proposed criteria for differentiating constrictive pericarditis from restrictive cardiomyopathy on the basis of the relative ratios of the left ventricular diastolic pressure, right ventricular diastolic pressure, and right ventricular systolic pressure. ${ }^{1020}$ Although there is a significant difference in the mean ratios between the two groups of patients, there is such overlap that these criteria are difficult to apply in an individual case (fig 3)..$^{5-7}$

The dynamic respiratory changes described above provide further diagnostic information at cardiac catheterisation. ${ }^{7}$ The dissociation of intrathoracic and intracardiac pressures can be analysed by using the left ventricular and 

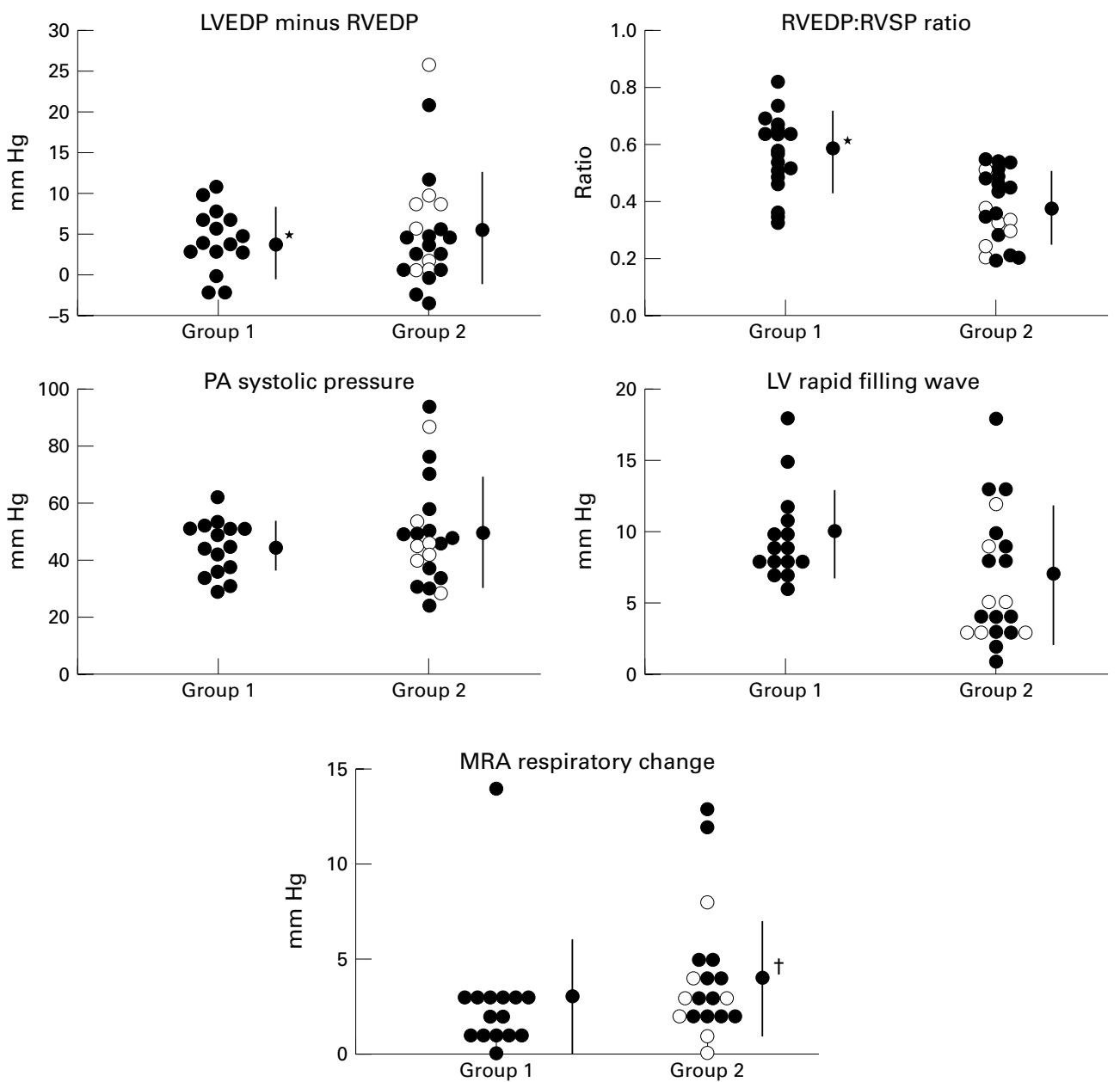

Figure 3 Conventional criteria at cardiac catheterisation. Group 1, patients with constrictive pericarditis; group 2, patients with restrictive cardiomyopathy or other types of cardiomyopathy and a normal pericardium. Although there are significant differences between the two groups, overlap makes it difficult to apply the criteria in an individual case. LVEDP, left ventricular end diastolic pressure; RVEDP, right ventricular end diastolic pressure; RVSP, right ventricular systolic pressure; $P A$, pulmonary artery; LV, left ventricular; MRA, mean right atrial pressure. Reproduced from Hurrell et al ${ }^{7}$ with permission of the American Heart Association.

pulmonary artery wedge pressure tracings obtained during phases of the respiratory cycle. In patients with constrictive pericarditis, there is a decrease in the initial wedge left ventricular pressure gradient during the first beat of inspiration. This change is not seen in patients with restrictive cardiomyopathy.

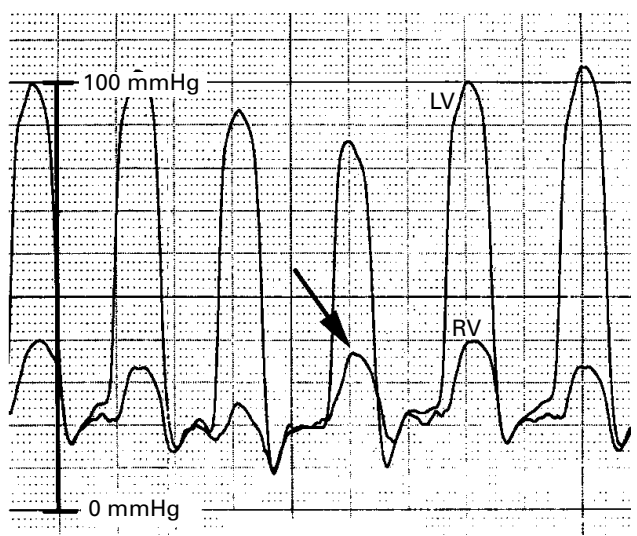

Figure 4 Pressures in the left (LV) and right ventricle (RV) of a patient with constrictive pericarditis. During peak inspiration (arrow), there is a decrease in $L V$ pressure and a concomitant increase in $R V$ pressure, indicating discordance of ventricular pressures.
The most useful information obtainable by cardiac catheterisation in the diagnosis of constrictive pericarditis pertains to the dynamic respiratory variation between the left and right ventricular pressure tracings. ${ }^{7}$ In patients with constrictive pericarditis, there is enhanced ventricular interaction, which is reflected in a discordance of the left and right ventricular pressures (fig 4). During peak inspiration, the left ventricular pressure decreases because there are decreases in the intrathoracic pressure and the filling of the left ventricle. As a result, the peak systolic pressure in the left ventricle decreases and there is an overall change in the contour of the curve, with a shortening of the width and a decrease in the entire area during systolic contraction. The enhanced ventricular interaction causes a compensatory increase in the filling of the right ventricle and an increase in the right sided stroke volume. During peak inspiration, this is seen as an increase in the peak pressure and systolic width of the right ventricular pressure curve. In patients with restrictive cardiomyopathy and a normal pericardium, there is a concordance of the left and right ventricular pressures (fig 5). The right and left ventricular systolic pressures decrease 


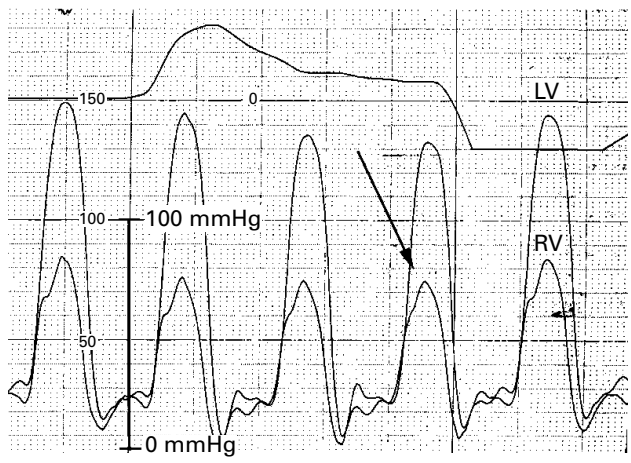

Figure 5 Pressures in the left $(L V)$ and right ventricle (RV) of a patient with restrictive cardiomyopathy. During peak inspiration (arrow) there is a decrease in LV pressure and a concomitant decrease in $R V$ pressure, indicating concordance of ventricular pressures.

during peak inspiration because of a decrease in intrathoracic pressure.

In a patient with constrictive pericarditis, the severity of the pericardial restraint is proportional to the degree of ventricular interaction seen on the peak inspiration beat. In patients with severe constriction, there is an obvious decrease in the area of the left ventricular pressure with a major decrease in systolic pressure; there is also a reciprocal increase in the area of the right ventricular pressure with an increase in systolic pressure. In less severe cases, there may be a subtle increase in the right ventricular systolic pressure and area during peak inspiration. Because the degree of enhanced ventricular interaction is proportional to the severity of pericardial restraint, it is useful to examine the relation between the left and right ventricular pressure curves if the patient has pericardial and myocardial disease. Some patients have a large degree of ventricular discordance during the respiratory cycle; these patients benefit most from pericardiectomy. Conversely, some patients have a mild degree of ventricular discordance but the severity of the diastolic pressure increase is disproportionate to the discordance. In these patients, the major pathophysiological process is a myocardial compliance abnormality; signs and symptoms of right sided heart failure remain if the pericardium is removed.

\section{The clinical challenge of constrictive pericarditis}

No single approach should be used to diagnose all cases of constrictive pericarditis. The diagnostic approach taken should be individualised for each patient. In some patients, the diagnosis may be made on the basis of the history, physical examination, and chest radiograph. In other patients, echocardiography, visualisation of the pericardium, and cardiac catheterisation all may be required. The most important diagnostic tool is the clinical suspicion of constrictive pericarditis in a patient with signs and symptoms of right sided heart failure that are disproportionate to pulmonary or left sided heart disease. Understanding the pathophysiology of this disease and using noninvasive and invasive techniques are helpful in diagnosis, particularly in the patient who has myocardial and pericardial disease.

1 Wood P. Chronic constrictive pericarditis. Am 7 Cardiol 1961;7:48-61.

2 Fowler NO. Constrictive pericarditis: its history and current status. Clin Cardiol 1995;18:341-50.

3 Ling LH, Oh JK, Schaff HV, et al. Constrictive pericarditis in the modern era: evolving clinical spectrum and impact on outcome after pericardiectomy. Circulation 1999;100: 1380-6.

4 Frank H, Globits S. Magnetic resonance imaging evaluation of myocardial and pericardial disease. 7 Magn Reson Imaging 1999;10:617-26.

5 Shabetai R. Controversial issues in restrictive cardiomyopathy. Postgrad Med f 1992;68(suppl 1):S47-51.

6 Vaitkus PT, Kussmaul WG. Constrictive pericarditis versus restrictive cardiomyopathy: a reappraisal and update of diagnostic criteria. Am Heart f 1991;122:1431-41.

7 Hurrell DG, Nishimura RA, Higano ST, et al. Value of Hurrell DG, Nishimura RA, Higano ST, et al. Value of
dynamic respiratory changes in left and right ventricular dynamic respiratory changes in left and right ventricular
pressures for the diagnosis of constrictive pericarditis. Cirpressures for the diagnosis
culation 1996;93:2007-13.

8 Oh JK, Hatle LK, Seward JB, et al. Diagnostic role of Doppler echocardiography in constrictive pericarditis. $\mathcal{f} \mathrm{Am}$ Coll Cardiol 1994;23:154-62.

9 Hatle LK, Appleton CP, Popp RL. Differentiation of constrictive pericarditis and restrictive cardiomyopathy by Doppler echocardiography. Circulation 1989;79:357-70.

10 Shabetai R, Fowler NO, Guntheroth WG. The hemodynamics of cardiac tamponade and constrictive pericarditis. Am $\mathcal{F}$ Cardiol 1970;26:480-9.

11 Siegel RJ, Shah PK, Fishbein MC. Idiopathic restrictive cardiomyopathy. Circulation 1984;70:165-9.

12 Ammash NM, Seward JB, Bailey KR, et al. Clinical profile and outcome of idiopathic restrictive cardiomyopathy. Circulation 2000;101:2490-6.

13 Himelman RB, Lee E, Schiller NB. Septal bounce, vena cava plethora, and pericardial adhesion: informative two-dimensional echocardiographic signs in the diagnosis of pericardial constriction. f Am Soc Echocardiogr 1988;1: of pericardic

14 Ling LH, Oh JK, Tei C, et al. Pericardial thickness measured with transesophageal echocardiography: feasibility and potential clinical usefulness. I Am Coll Cardiol 1997;29: 1317-23.

15 Nishimura RA, Tajik AJ. Evaluation of diastolic filling of left ventricle in health and disease: Doppler echocardiography is the clinician's Rosetta stone. $\mathcal{F}$ Am Coll Cardiol 1997;30: $8-18$.

16 Oh JK, Tajik AJ, Appleton CP, et al. Preload reduction to unmask the characteristic Doppler features of constrictive
pericarditis: a new observation. Circulation 1997;95:796-9.

17 Masui T, Finck S, Higgins CB. Constrictive pericarditis and restrictive cardiomyopathy: evaluation with MR imaging. Radiology 1992;182:369-73.

18 Breen JF. Imaging of the pericardium. 7 Thorac Imaging 2001;16:47-54.

19 Connolly DC, Wood EH. Cardiac catheterization in heart failure and cardiac constriction. Trans Am Coll Cardiol failure and cardiac

20 Meaney E, Shabetai R, Bhargava V, et al. Cardiac amyloidosis, constrictive pericarditis and restrictive cardiomyopathy. Am f Cardiol 1976;38:547-56. 